Homology, Homotopy and Applications, vol.23(2), 2021, pp.59-68

\title{
A REMARK ON THE DOUBLE COMPLEX OF A COVERING FOR SINGULAR COHOMOLOGY
}

\author{
ROBERTO FRIGERIO AND ANDREA MAFFEI
}

(communicated by Donald M. Davis)

\begin{abstract}
Given an open covering of a paracompact topological space $X$, there are two natural ways to construct a map from the cohomology of the nerve of the covering to the cohomology of $X$. One of them is based on a partition of unity, and is more topological in nature, while the other one relies on the double complex associated to an open covering, and has a more algebraic flavour. In this paper we prove that these two maps coincide.
\end{abstract}

\section{Introduction}

Let $X$ be a paracompact space, and let $\mathcal{U}=\left\{U_{i}\right\}_{i \in I}$ be an open covering of $X$. We denote by $N(\mathcal{U})$ the nerve of $\mathcal{U}$, i.e. the simplicial set having $I$ as set of vertices, in which a finite subset $\left\{i_{0}, \ldots, i_{k}\right\} \subseteq I$ spans a simplex if and only if $U_{i_{0}} \cap \ldots \cap U_{i_{k}} \neq$ $\varnothing$. As usual, we endow the geometric realization $|N(\mathcal{U})|$ of $N(\mathcal{U})$ with the weak topology associated to the natural $\mathrm{CW}$ structure of $|N(\mathcal{U})|$.

Any partition of unity $\Phi=\left\{\varphi_{i}: X \rightarrow \mathbb{R}\right\}_{i \in I}$ subordinate to $\mathcal{U}$ induces a map

$$
f_{\Phi}: X \rightarrow|N(\mathcal{U})|, \quad f_{\Phi}(x)=\sum_{i \in I} \varphi_{i}(x) \cdot i
$$

Moreover, the homotopy class of $f_{\Phi}$ does not depend on the chosen partition of unity $\Phi$. Indeed, if $\Psi$ is another partition of unity, then we have a well-defined homotopy $t f_{\Psi}+(1-t) f_{\Phi}$ between $f_{\Psi}$ and $f_{\Phi}$. Therefore, if $R$ is any ring with unity, the map $f_{\Phi}$ induces a map

$$
f^{*}=f_{\Phi}^{*}: H^{*}(|N(\mathcal{U})|, R) \rightarrow H^{*}(X, R),
$$

which does not depend on the choice of $\Phi$. Throughout this paper, we fix a ring with unity $R$, and for any topological space $Y$ we denote by $C^{*}(Y)=C^{*}(Y, R)$ (resp. $H^{*}(Y)=H^{*}(Y, R)$ ) the singular cochain complex (resp. the singular cohomology algebra) of $Y$ with coefficients in $R$.

There is another natural way to define a map from the (simplicial) cohomology of $N(\mathcal{U})$ to the singular cohomology of $X$, using a double complex associated to the covering $\mathcal{U}$. The idea of this construction goes back at least to the paper of Weil on the de Rham Theorem [Wei52], where the author dealt with differential forms in

Received March 7, 2020, revised July 2, 2020; published on April 7, 2021.

2010 Mathematics Subject Classification: 55N10, 55N05, 55T99.

Key words and phrases: nerve of a covering, double complex associated to an open covering.

Article available at http://dx.doi.org/10.4310/HHA.2021.v23.n2.a4

Copyright (C) 2021, Roberto Frigerio and Andrea Maffei. Permission to copy for private use granted. 
Section 2, and with singular chains in Section 3. Let $C^{* * *}(\mathcal{U})$ be the double complex associated to the covering $\mathcal{U}$, i.e. for every $(p, q) \in \mathbb{N}^{2}$ let

$$
C^{p, q}(\mathcal{U})=\prod_{\underline{i} \in I_{p}} C^{q}\left(U_{\underline{i}}\right)
$$

where $I_{p}$ denotes the set of ordered $(p+1)$-tuples $\left(i_{0}, \ldots, i_{p}\right) \in I^{p+1}$ such that $U_{\underline{i}}:=$ $U_{i_{0}} \cap \ldots \cap U_{i_{p}} \neq \varnothing$ (in particular, $I_{0}=\left\{i \in I \mid U_{i} \neq \varnothing\right\}$ ). We refer the reader to Section 2 for the precise definition of this double complex.

To the double complex $C^{* * *}(\mathcal{U})$ there is associated the total complex $T^{*}$, and we have maps

$$
\alpha_{X}: H^{*}(X) \rightarrow H^{*}\left(T^{*}\right), \quad \beta: H^{*}(N(\mathcal{U})) \rightarrow H^{*}\left(T^{*}\right)
$$

from the singular cohomology of $X$ to the cohomology of $T^{*}$ and from the simplicial cohomology of $N(\mathcal{U})$ to the cohomology of $T^{*}$. Moreover, the map $\alpha$ turns out to be an isomorphism (see Section 2).

Let now $\nu: H^{*}(|N(\mathcal{U})|) \rightarrow H^{*}(N(\mathcal{U}))$ be the canonical isomorphism between the simplicial cohomology of $N(\mathcal{U})$ and the singular cohomology of its geometric realization (see Section 3). By setting $\eta=\alpha_{X}^{-1} \circ \beta \circ \nu$ we have thus defined a map

$$
\eta: H^{*}(|N(\mathcal{U})|) \rightarrow H^{*}(X) .
$$

The main result of this paper shows that the maps $f^{*}$ and $\eta$ coincide:

Theorem 1.1. The maps

$$
f^{*}: H^{*}(|N(\mathcal{U})|) \rightarrow H^{*}(X), \quad \eta: H^{*}(|N(\mathcal{U})|) \rightarrow H^{*}(X)
$$

coincide.

We were motivated to study whether the maps $f^{*}$ and $\eta$ should coincide by the fact that our result may be exploited to provide an interpretation of a result by Ivanov on the bounded cohomology of topological spaces. In his pioneering paper [Gro82], Gromov introduced the notion of bounded singular cochains. For any topological space $X$, bounded cochains (with real coefficients) provide a subcomplex $C_{b}^{*}(X)$ of $C^{*}(X)$. The cohomology of the complex $C_{b}^{*}(X)$ is denoted by $H_{b}^{*}(X)$, and defines the bounded cohomology of $X$. The inclusion of bounded cochains into ordinary cochains induces the comparison map $c^{*}: H_{b}^{*}(X) \rightarrow H^{*}(X)$.

A subset $U$ of $X$ is amenable if, for every $x_{0} \in U$, the image of the map $\pi_{1}\left(U, x_{0}\right) \rightarrow$ $\pi_{1}\left(X, x_{0}\right)$ induced by the inclusion is amenable, and a covering of $X$ is amenable if each of its elements is amenable. Gromov's Vanishing Theorem [Gro82] asserts that, if $X$ admits an open amenable covering $\mathcal{U}$ of multiplicity $n$, then the comparison map $c^{m}: H_{b}^{m}(X) \rightarrow H^{m}(X)$ is null for every $m \geqslant n$ (if $X$ is a closed $n$-dimensional manifold, then the vanishing of the comparison map in degree $n$ has strong implications on the vanishing of several interesting invariants of $X$, such as the simplicial volume, the minimal volume, the volume entropy).

Gromov's Vanishing Theorem was generalized and made more precise by Ivanov in [Iva87, Iva], where it is shown that, if $\mathcal{U}$ is a nice covering of $X$, then there exists 
a map $\theta^{*}: H_{b}^{*}(X) \rightarrow H_{b}^{*}(|N(\mathcal{U})|)$ which makes the following diagram commute

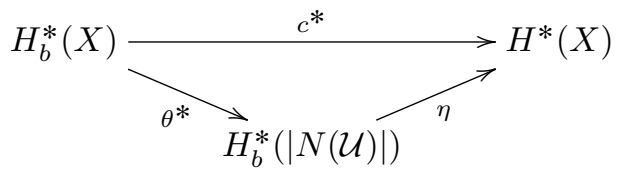

(we refer the reader to [Iva] for the definition of nice covering; for example, any open covering of a hereditary paracompact space is nice).

Our result implies that, in the diagram above, the map $\eta$ can be replaced by the map $f^{*}$. This provides a clear topological interpretation of the well-known concept that amenable spaces are somewhat invisible to bounded cohomology: in fact, the map $f: X \rightarrow|N(\mathcal{U})|$ shrinks each element of $\mathcal{U}$ into a contractible subset of $|N(\mathcal{U})|$, thus trivializing its topology, while the commutativity of the diagram implies that the information carried by $|N(\mathcal{U})|$ still suffices to completely determine singular cohomology classes admitting a bounded representative. For recent alternative proofs of the fact that the comparison map $c^{*}$ factors through $f^{*}$ (which, however, do not deal with the question whether $f^{*}=\eta$ in general) we refer the reader to [FM, Chapter 6] and $[\mathbf{L S}]$.

\section{The double complex associated to an open covering}

Let $\mathcal{U}=\left\{U_{i}\right\}_{i \in I}$ be an open covering of the topological space $X$. We now thoroughly describe the horizontal and the vertical differentials of the double complex $C^{*, *}(\mathcal{U})$ defined in the introduction, also fixing the notation we will need later.

If $\varphi \in C^{p, q}(\mathcal{U})$ and $\underline{i} \in I_{p}$, then we denote by $\varphi_{\underline{i}}$ the projection of $\varphi$ on $C^{q}\left(U_{\underline{i}}\right)$. For every $(p, q) \in \mathbb{N}^{2}$ we denote by

$$
\delta_{v}^{p, q}: C^{p, q}(\mathcal{U}) \rightarrow C^{p, q+1}(\mathcal{U})
$$

the "vertical" differential which restricts to the usual differential $C^{q}\left(U_{\underline{i}}\right) \rightarrow C^{q+1}\left(U_{\underline{i}}\right)$ for every $\underline{i} \in I_{p}$, and by

$$
\delta_{h}^{p, q}: C^{p, q}(\mathcal{U}) \rightarrow C^{p+1, q}(\mathcal{U})
$$

the "horizontal" differential such that, for every $\underline{i}=\left(i_{0}, \ldots, i_{p+1}\right) \in I_{p+1}$ and every $\varphi \in C^{p, q}(\mathcal{U})$,

$$
\left(\delta_{h}^{p, q}(\varphi)\right)_{\underline{i}}=\left.\sum_{k=0}^{p+1}(-1)^{k}\left(\varphi_{\left(i_{0}, \ldots, \hat{i}_{k}, \ldots, i_{p+1}\right)}\right)\right|_{U_{\underline{i}}} .
$$

We augment the double complex $C^{*, *}(\mathcal{U})$ as follows. We define $C_{q}^{\mathcal{U}}$ as the subcomplex of the singular chain complex $C_{q}(X)$ generated (over $R$ ) by those singular simplices $s: \Delta^{q} \rightarrow X$ such that $s\left(\Delta^{q}\right)$ is contained in $U_{i}$ for some $i \in I$. We then set $C^{-1, q}(\mathcal{U})=C_{\mathcal{U}}^{q}=\operatorname{Hom}\left(C_{q}^{\mathcal{U}}, R\right)$. The usual boundary maps of the complex $C_{*}^{\mathcal{U}}$ induce dual coboundary maps, which endow $C_{\mathcal{U}}^{*}$ with the structure of a complex. The inclusion of the complex $C_{*}^{\mathcal{U}}$ in the full complex of singular chains induces a map of complexes $\tilde{\gamma}: C^{*}(X) \rightarrow C_{\mathcal{U}}^{*}$. It is known that the map $\gamma$ induced in cohomology is an isomorphism (see e.g. [Hat02, Proposition 2.21]) and we will identify the singular cohomology of $X$ with the cohomology of the complex $C_{\mathcal{U}}^{q}$ via $\gamma$. The augmentation 
maps $\delta^{-1, q}: C^{-1, q}(\mathcal{U}) \rightarrow C^{0, q}(\mathcal{U})$ are defined by setting, for every $i \in I_{0}$,

$$
\left(\delta^{-1, q}(\varphi)\right)_{i}=\left.\varphi\right|_{U_{i}} .
$$

In order to define the augmentation of the vertical complexes, we consider the Cech complex given by $C^{p,-1}(\mathcal{U})=\check{C}^{p}(\mathcal{U})=\prod_{\underline{i \in I_{p}}} R$, with boundary maps defined as in formula (1). We then define the augmentation maps $\delta^{p,-1}: C^{p,-1}(\mathcal{U}) \rightarrow C^{p, 0}(\mathcal{U})$ by setting

$$
\left(\delta^{p,-1}(\varphi)\right)_{\underline{i}}(s)=\varphi_{\underline{i}} \in R
$$

for every $\varphi \in C^{p,-1}(\mathcal{U})$, every $\underline{i}=\left(i_{0}, \ldots, i_{p}\right) \in I_{p}$ and every singular simplex $s: \Delta^{0} \rightarrow$ $U_{i_{0}} \cap \ldots \cap U_{i_{p}}$.

Remark 2.1. The complex $\check{C}^{*}(\mathcal{U})$ computes the Cech cohomology of the covering $\mathcal{U}$ with coefficients in the constant presheaf $R$. Such cohomology, which is usually denoted by $\breve{H}(\mathcal{U})$, is tautologically isomorphic to the simplicial cohomology of the nerve $N(\mathcal{U})$. It is costumary to rather study the Cech cohomology of $\mathcal{U}$ with coefficients in the locally constant sheaf $R$. However this cohomology does not always coincide with the cohomology of $N(\mathcal{U})$. They coincide, for example, under the assumption that every $U_{\underline{i}}, i \in I_{p}, p \in \mathbb{N}$, is path connected.

It is well known that, for every $q \in \mathbb{N}$, the row

$$
0 \longrightarrow C^{-1, q}(\mathcal{U}) \stackrel{\delta_{h}^{-1, q}}{\longrightarrow} C^{0, q}(\mathcal{U}) \stackrel{\delta_{h}^{0, q}}{\longrightarrow} \cdots \stackrel{\delta_{h}^{p-1, q}}{\longrightarrow} C^{p, q}(\mathcal{U}) \stackrel{\delta_{h}^{p, q}}{\longrightarrow} \cdots
$$

of the augmented double complex introduced above is exact. A proof of the analogous statement for singular chains can be found, for example, in [BT82, Proposition 15.2], and the statement for cochains follows immediately.

As a consequence, the cohomology groups of the complex $C^{-1, *}$ are isomorphic to the cohomology of the total complex $T^{*}$ associated to the double complex. Recall that $T^{*}$ is defined by setting

$$
T^{n}=\bigoplus_{\substack{(p, q) \in \mathbb{N}^{2} \\ p+q=n}} C^{p, q}(\mathcal{U})
$$

with differential $\delta^{n}: T^{n} \rightarrow T^{n+1}$ given by $\delta^{n}=\bigoplus_{p+q=n}\left(\delta_{h}^{p, q}+(-1)^{p} \delta_{v}^{p, q}\right)$. The augmentation maps induce morphisms of complexes $\widetilde{\alpha}^{*}: C_{\mathcal{U}}^{*} \rightarrow T^{*}$ and $\widetilde{\beta}^{*}: \check{C}^{*} \rightarrow T^{*}$ and we denote by $\alpha, \beta$ the maps induced by $\alpha^{*}, \beta^{*}$ on cohomology. Since the rows (2) are exact, $\alpha$ is an isomorphism in every degree and the map $\alpha \circ \gamma: H^{*}(X) \rightarrow H^{*}\left(T^{*}\right)$ is the isomorphism $\alpha_{X}$ defined in the introduction. We define $\zeta=\alpha^{-1} \circ \beta$ and $\eta=$ $\alpha_{X}^{-1} \circ \beta \circ \nu$.

The notation introduced so far is summarized in the following diagram:

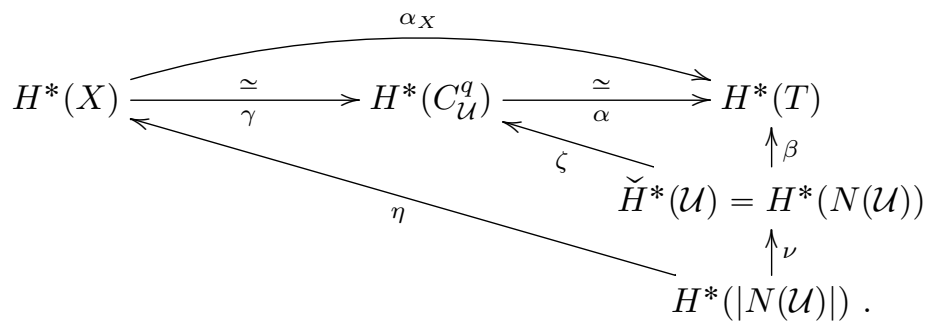


When we want to stress the dependence of these constructions on the covering $\mathcal{U}$ we write $\alpha_{\mathcal{U}}, \beta_{\mathcal{U}}$, etc.

\section{The case of a simplicial complex}

In this section we analyze the double complex associated to the open star covering of the geometric realization $X=|S|$ of a simplicial complex $S$. Let $I$ be the vertex set of $S$. We consider the open covering $\mathcal{U}^{*}=\left\{U_{i}^{*}\right\}_{i \in I}$ of $|S|$ given by the open stars of the vertices, i.e., for every $i \in I$ we set $U_{i}=\left\{x \in|S|: x_{i}>0\right\}$, where $x_{i}$ denotes the barycentric coordinate of the point $x$ relative to the vertex $i$. Observe that the simplical complexes $N\left(\mathcal{U}^{*}\right)$ and $S$ on the set of vertices $I$ are equal and we will identify them. Hence, in this case $\eta_{\mathcal{U}^{*}}: H^{*}(|S|) \rightarrow H^{*}(|S|)$. Notice also that in this case all intersections $U_{\underline{i}}^{*}$ are contractible, hence, also the columns of the augmented double complex are exact. As a consequence, $\beta$ and $\zeta$ are isomorphisms. The next proposition shows that the map $\eta$ is the identity in this case.

Proposition 3.1. If $S$ is a simplicial complex and $\mathcal{U}^{*}$ is the covering described above then $\eta=I d$.

To prove this proposition we will perform a computation by describing a lift of $\zeta$ at the level of cochains. To simplify the computations we will use alternating cochains, whose definition is recalled below.

\section{Construction of $\widetilde{\zeta}$}

We start by describing a lift

$$
\widetilde{\zeta}: \check{C}(\mathcal{U}) \rightarrow C^{-1, p}(\mathcal{U})=C_{\mathcal{U}}^{p}
$$

of the map $\zeta$ at the level of cochains. We first construct chain homotopies

$$
K^{p, q}: C^{p, q}(\mathcal{U}) \rightarrow C^{p-1, q}(\mathcal{U}), \quad p \geqslant 0, \quad q \geqslant 0 .
$$

For each singular simplex $s$ with image contained in some open subset $U_{i}$ we fix an index $i(s)$ such that $\operatorname{Im} s \subseteq U_{i(s)}$. For all $\varphi \in C^{p, q}(\mathcal{U})$ and for all singular simplices $s$ with image contained in $U_{\underline{i}}$ for some $\underline{i} \in I_{p-1}, p \geqslant 0$, we define

$$
\left(K^{p, q}(\varphi)_{\underline{i}}\right)(s)=\varphi_{i(s), \underline{i}}(s)
$$

(when $p=0$ there is no index $\underline{i}$ and we just take $s \in C_{q}^{\mathcal{U}}$ ). It is easy to check that $\delta_{h}^{p-1, q} K^{p, q}+K^{p+1, q} \delta_{h}^{p, q}=$ Id for every $p \geqslant 0, q \geqslant 0$. Hence, if we define

$$
\widetilde{\zeta}=(-1)^{\frac{p(p+1)}{2}} K^{0, p} \circ \delta_{v}^{0, p-1} \circ K^{1, p-1} \circ \cdots \circ K^{p-1,1} \circ \delta_{v}^{p-1,0} \circ K^{p, 0} \circ \delta_{v}^{p,-1}
$$

then for every cocycle $\varphi \in \check{C}^{p}(\mathcal{U})$ we have $\zeta([\varphi])=[\widetilde{\zeta}(\varphi)]$ in $H^{p}\left(C_{\mathcal{U}}^{*}\right)$.

\section{Singular and algebraic simplices}

Let us now recall the construction of the isomorphism $\nu$ between the simplicial cohomology $H^{*}(S)$ of $S$ and the singular cohomology $H^{*}(|S|)$ of its geometric realization. Let $C_{*}(S)$ be the chain complex of simplicial chains on $S$, i.e. let $C_{p}$ be the free 
$R$-module with basis

$$
\left\{\left(i_{0}, \ldots, i_{p}\right) \in I^{p+1} \mid\left\{i_{0}, \ldots, i_{p}\right\} \text { is a simplex of } S\right\},
$$

and let $C^{*}(S)$ be the dual chain complex of $C_{*}(S)$. Elements of the basis just described are usually called algebraic simplices.

For any algebraic simplex $\sigma=\left(i_{0}, \ldots, i_{p}\right)$ of $S$, one defines the singular simplex $\langle\sigma\rangle: \Delta^{p} \rightarrow|S|$ by setting

$$
\langle\sigma\rangle\left(t_{0}, \ldots, t_{p}\right)=t_{0} i_{0}+\cdots+t_{p} i_{p} .
$$

The map $\sigma \mapsto\langle\sigma\rangle$ extends to a chain map $C_{*}(S) \rightarrow C_{*}(|S|)$, whose dual map $\widetilde{\nu}: C^{*}(|S|) \rightarrow C^{*}(S)$ induces the isomorphism $\nu: H^{*}(|S|) \rightarrow H^{*}(S)$ (see e.g. [Hat02] Theorem 2.27). We write $\nu_{S}, \widetilde{\nu}_{S}$ when we want to stress the dependence on the simplicial complex.

\section{Alternating cochains}

To compute $\zeta \circ \nu$ it is convenient to use alternating cochains. Let $\mathfrak{S}_{p+1}$ be the permutation group of $\{0, \ldots, p\}$. We say that a simplicial cochain $\varphi \in C^{p}(S)$ is alternating if $\varphi\left(i_{\tau(0)}, \ldots, i_{\tau(p)}\right)=\varepsilon(\tau) \varphi\left(i_{0}, \ldots, i_{p}\right)$ for every $\tau \in \mathfrak{S}_{p+1}$, and $\varphi\left(i_{0}, \ldots, i_{p}\right)=0$ whenever $i_{j}=i_{j^{\prime}}$ for some $j \neq j^{\prime}$. Alternating cochains form a subcomplex of the complex of cochains which is homotopy equivalent to the full complex (see e.g. [ES52, Chapter VI, Section 6, Theorems 6.9 and 6.10]).

Alternating cochains may be defined also in the context of singular homology as follows. For every $\tau \in \mathfrak{S}_{p+1}$ denote by $\rho_{\tau}: \Delta^{p} \rightarrow \Delta^{p}$ the affine automorphism of $\Delta^{p}$ defined by $\rho_{\tau}\left(t_{0}, \ldots, t_{p}\right)=\left(t_{\tau(0)}, \ldots, t_{\tau(p)}\right)$. If $X$ is a topological space, we say that a singular cochain $\varphi \in C^{p}(X)$ is alternating if $\varphi\left(s \circ \rho_{\tau}\right)=\varepsilon(\tau) \varphi(s)$ for every $\tau \in \mathfrak{S}_{p+1}$ and every singular simplex $s: \Delta^{p} \rightarrow X$, and $\varphi(s)=0$ for every singular simplex $s$ such that $s=s \circ \rho_{\tau}$ for an odd permutation $\tau \in \mathfrak{S}_{p+1}$. Alternating singular cochains form a subcomplex of the complex of singular cochains which is homotopy equivalent to the full complex (see e.g. [Bar95]). Moreover, the map $\widetilde{\nu}$ introduced above sends alternating singular cochains to alternating simplicial cochains, and both the homotopy maps $K^{p, q}$ and the vertical differential send alternating cochains to alternating ones.

We want to compute $\widetilde{\zeta}(\varphi)$ on singular simplices of the form $\langle\sigma\rangle$, as $\sigma$ varies among the algebraic simplices of $S$. However, simplices of $S$ are not contained in any $U_{i}^{*}$. We will then make use of the barycentric subdivision $S^{\prime}$ of $S$, together with a suitable simplicial approximation of the identity $S^{\prime} \rightarrow S$. Let $I^{\prime}$ be the set of vertices of $S^{\prime}$. This set is in bijective correspondence with the set of simplices of $S$ : for $i^{\prime} \in I^{\prime}$ we denote by $\Delta_{i^{\prime}}$ the simplex of $S$ of which $i^{\prime}$ is the barycenter; in the opposite direction, if $\Delta$ is a simplex of $S$ we denote by $i_{\Delta}^{\prime}$ its barycenter. The $p$-simplices of $S^{\prime}$ are then the subsets $\left\{i_{0}^{\prime}, \ldots, i_{p}^{\prime}\right\}$ where $\Delta_{i_{0}^{\prime}} \subset \cdots \subset \Delta_{i_{p}^{\prime}}$.

If for every simplex $\Delta$ of $S$ we denote by $b_{\Delta} \in|S|$ the geometric barycenter of $\Delta$ then the map $b:\left|S^{\prime}\right| \rightarrow|S|$ defined by $b\left(\sum_{\Delta} t_{\Delta} i_{\Delta}^{\prime}\right)=\Sigma_{\Delta} t_{\Delta} b_{\Delta}$ is a homeomorphism, and we will identify the geometric realizations of $S^{\prime}$ and $S$ via this map. We construct a second map from $\left|S^{\prime}\right|$ to $|S|$ as follows. We fix an auxiliary total ordering on $I$, and we define a simplicial map $g: S^{\prime} \rightarrow S$ by setting

$$
g\left(i^{\prime}\right)=\max \Delta_{i^{\prime}}
$$


for every vertex $i^{\prime}$ of $S^{\prime}$. The geometric realization $|g|:|S|=\left|S^{\prime}\right| \rightarrow|S|$ of $g$ is homotopic to $b$ via the homotopy $t b+(1-t)|g|, t \in[0,1]$.

We may define the map $i$ used to construct the homotopies $K^{p, q}$ in such a way that, for every algebraic simplex $\sigma^{\prime}=\left(i_{0}^{\prime}, \ldots, i_{p}^{\prime}\right)$ of $C_{*}\left(S^{\prime}\right)$,

$$
i\left(\left\langle\sigma^{\prime}\right\rangle\right)=\min \left\{g\left(i_{0}^{\prime}\right), \ldots, g\left(i_{p}^{\prime}\right)\right\} .
$$

For simplicity, we will denote $i\left(\left\langle\sigma^{\prime}\right\rangle\right)$ by $i\left(\sigma^{\prime}\right)$. With this choice, the singular simplex $\left\langle\sigma^{\prime}\right\rangle$ is supported in $U_{i\left(\sigma^{\prime}\right)}^{*}$ as required in the definition of the map $i$.

Let $\alpha=\left(\alpha_{\underline{i}}\right) \in C^{h, k}\left(\mathcal{U}^{*}\right)$ and let $\sigma^{\prime}=\left(i_{0}^{\prime}, \ldots, i_{k+1}^{\prime}\right) \in C_{k+1}\left(U_{i}^{*}\right), \underline{i} \in I_{h}$, be an algebraic $(k+1)$-simplex of $S^{\prime}$. If $\partial_{h} \sigma^{\prime}=\left(i_{0}^{\prime}, \ldots, \hat{i}_{h}^{\prime}, \ldots, i_{k+1}^{\prime}\right)$ denotes the algebraic $h$-th face of $\sigma^{\prime}$, then

$$
\left(\delta_{v}^{h-1, k} K^{h, k}(\alpha)\right)\left(\left\langle\sigma^{\prime}\right\rangle\right)=\sum_{h=0}^{k+1}(-1)^{h}\left(K^{h, k}(\alpha)\right)_{\underline{i}}\left(\left\langle\partial_{h} \sigma^{\prime}\right\rangle\right)=\sum_{h=0}^{k+1}(-1)^{h} \alpha_{i\left(\partial_{h} \sigma^{\prime}\right), \underline{i}}\left(\left\langle\partial_{h} \sigma^{\prime}\right\rangle\right) .
$$

Lemma 3.2. Let $\varphi$ be an alternating cocycle in $C^{p}\left(N\left(\mathcal{U}^{*}\right)\right)=\check{C}^{p}\left(\mathcal{U}^{*}\right)$, and let $\sigma^{\prime} \in$ $C_{p}\left(S^{\prime}\right)$ be an algebraic simplex. Then

$$
(\widetilde{\zeta}(\varphi))\left(\left\langle\sigma^{\prime}\right\rangle\right)=\varphi\left(g_{*}\left(\sigma^{\prime}\right)\right)
$$

where $g_{*}: C_{p}\left(S^{\prime}\right) \rightarrow C_{p}(S)$ is the map induced by $g: S^{\prime} \rightarrow S$.

Proof. Let $\sigma^{\prime}=\left(i_{0}^{\prime}, \ldots, i_{p}^{\prime}\right)$ and set $\Delta_{\ell}=\Delta_{i_{\ell}^{\prime}}$ for $\ell=0, \ldots, p$ and $i_{\ell}=g\left(i_{\ell}^{\prime}\right)$. Recall that simplices of $S^{\prime}$ correspond to comparable subsets of a simplex of $S$. Moreover, since $\varphi$ is alternating, both $g^{*}(\varphi)$ and $\widetilde{\zeta}(\varphi)$ are alternating, thus in order to check that the equality of the statement holds we may assume that

$$
\Delta_{0} \subsetneq \Delta_{1} \cdots \subsetneq \Delta_{p}
$$

By definition we have $i_{\ell}=\max \Delta_{\ell}$, hence in particular $i_{0} \leqslant i_{1} \leqslant \cdots \leqslant i_{p}$. Since $\varphi$ is alternating, this implies at once that

$$
\varphi\left(g_{*}\left(\sigma^{\prime}\right)\right)= \begin{cases}\varphi_{i_{0}, i_{1}, \ldots, i_{p}} & \text { if } i_{0}<\cdots<i_{p} \\ 0 & \text { otherwise }\end{cases}
$$

Let us now compute $(\widetilde{\zeta}(\varphi))\left(\left\langle\sigma^{\prime}\right\rangle\right)$. For every algebraic simplex $\tau_{k}^{\prime} \in C_{k}\left(S^{\prime}\right)$, we write $\tau_{k-1}^{\prime}<\tau_{k}^{\prime}$ if $\tau_{k-1}^{\prime}$ is an algebraic face of $\tau_{k}^{\prime}$, i.e. if there exists $h=0, \ldots, k$ such that $\tau_{k-1}^{\prime}=\partial_{h} \tau_{k}^{\prime}$. By iterating (3) we get

$$
(\widetilde{\zeta}(\varphi))\left(\left\langle\sigma^{\prime}\right\rangle\right)=(-1)^{\frac{p(p+1)}{2}} \sum_{\sigma_{0}^{\prime}<\cdots<\sigma_{p}^{\prime}=\sigma^{\prime}} \pm \varphi_{i\left(\sigma_{0}^{\prime}\right), i\left(\sigma_{1}^{\prime}\right), \ldots, i\left(\sigma_{p}^{\prime}\right)} .
$$

Let now $\sigma_{0}^{\prime}<\cdots<\sigma_{p}^{\prime}$ be a fixed descending sequence of faces of $\sigma^{\prime}$. Since the map $i$ is given by taking a minimum we have $i\left(\sigma_{0}^{\prime}\right) \geqslant i\left(\sigma_{1}^{\prime}\right) \geqslant \cdots \geqslant i\left(\sigma_{p}^{\prime}\right)$ and all these elements

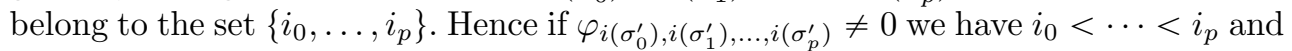
$i\left(\sigma_{\ell}^{\prime}\right)=i_{p-\ell}$ for every $\ell$. In particular $(\widetilde{\zeta}(\varphi))\left(\left\langle\sigma^{\prime}\right\rangle\right)$ agrees with $\varphi\left(g_{*}\left(\sigma^{\prime}\right)\right)$ in the second case of formula (4). 
Assume now $i_{0}<\cdots<i_{p}$. As just observed, if $\varphi_{i\left(\sigma_{0}^{\prime}\right), i\left(\sigma_{1}^{\prime}\right), \ldots, i\left(\sigma_{p}^{\prime}\right)} \neq 0$ then $i\left(\sigma_{\ell}^{\prime}\right)=$ $i_{p-\ell}$ for every $\ell$, and this readily implies that the unique non-trivial addend in the right-hand sum in (5) corresponds to the sequence

$$
\bar{\sigma}_{0}^{\prime}=\left(i_{p}^{\prime}\right), \quad \bar{\sigma}_{1}^{\prime}=\left(i_{p-1}^{\prime}, i_{p}^{\prime}\right), \quad \ldots \quad, \bar{\sigma}_{p}^{\prime}=\left(i_{0}^{\prime}, \ldots, i_{p-1}^{\prime}, i_{p}^{\prime}\right) .
$$

In particular, for every $j=0, \ldots, p-1$ we have $\bar{\sigma}_{j}^{\prime}=(-1)^{0} \partial_{0} \bar{\sigma}_{j+1}^{\prime}$. Hence

$$
(\widetilde{\zeta}(\varphi))\left(\left\langle\sigma^{\prime}\right\rangle\right)=(-1)^{\frac{p(p+1)}{2}} \varphi_{i\left(\bar{\sigma}_{0}^{\prime}\right), i\left(\bar{\sigma}_{1}^{\prime}\right), \ldots, i\left(\bar{\sigma}_{p}^{\prime}\right)}=(-1)^{\frac{p(p+1)}{2}} \varphi_{i_{p}, i_{p-1}, \ldots, i_{0}}=\varphi_{i_{0}, i_{1}, \ldots, i_{p}}
$$

settling also the first case in formula (4).

Before proving the proposition we notice that the map $C_{*}(S) \rightarrow C_{*}(|S|)$ constructed above does not factor through $C_{*}^{\mathcal{U}^{*}}$ because no positive-dimensional simplex of $S$ is contained in $U_{i}^{*}$ for any $i \in I$. However the analogous map from $C_{*}\left(S^{\prime}\right)$ to $C_{*}(|S|)$ does. Hence the map $\widetilde{\nu}_{S^{\prime}}: C^{*}\left(\left|S^{\prime}\right|\right) \rightarrow C^{*}\left(S^{\prime}\right)$ factors as $\widetilde{\nu}_{S^{\prime}}=\tilde{\mu} \circ \widetilde{\gamma}$, where $\tilde{\gamma}: C^{*}\left(\left|S^{\prime}\right|\right) \rightarrow C_{\mathcal{U}^{*}}^{*}$ is the map defined in Section 2 , and $\tilde{\mu}: C_{\mathcal{U}^{*}}^{*} \rightarrow C^{*}\left(S^{\prime}\right)$. We denote by $\mu: H^{*}\left(C_{\mathcal{U}^{*}}^{*}\right) \rightarrow H^{*}(S)$ the map induced by $\tilde{\mu}$ on cohomology.

Proof of Proposition 3.1. Being $\nu_{S^{\prime}}: H^{*}(|S|)=H^{*}\left(\left|S^{\prime}\right|\right) \rightarrow H^{*}\left(S^{\prime}\right)$ injective and $|g|$ homotopic to the identity, in order to prove the proposition it is sufficient to show that $\nu_{S^{\prime}} \circ \eta=\nu_{S^{\prime}} \circ|g|^{*}$. Now recall that $\eta=\gamma^{-1} \circ \zeta \circ \nu_{S}$, hence $\nu_{S^{\prime}} \circ \eta=\mu \circ \zeta \circ \nu_{S}$. Hence it is enough to prove that $\mu\left(\zeta\left(\nu_{S}(c)\right)\right)=\nu_{S^{\prime}}\left(|g|^{*}(c)\right)$ for all $c \in H^{p}(|S|)$ or, equivalently, that

$$
\widetilde{\mu}\left(\widetilde{\zeta}\left(\widetilde{\nu}_{S}(\psi)\right)\right)\left(\sigma^{\prime}\right)=\widetilde{\nu}_{S^{\prime}}\left(|g|^{*}(\psi)\right)\left(\sigma^{\prime}\right),
$$

where $\psi \in C^{p}(|S|)$ is a cocycle and $\sigma^{\prime}$ is any algebraic simplex of $S^{\prime}$. Moreover, as observed above we can choose $\psi$ to be alternating. However, if we set $\phi=\widetilde{\nu}_{S}(\psi)$, then

$$
\begin{gathered}
\mu\left(\widetilde{\zeta}\left(\widetilde{\nu}_{S}(\psi)\right)\right)\left(\sigma^{\prime}\right)=\mu(\widetilde{\zeta}(\phi))\left(\sigma^{\prime}\right)=(\widetilde{\zeta}(\varphi))\left(\left\langle\sigma^{\prime}\right\rangle\right), \\
\widetilde{\nu}_{S^{\prime}}\left(|g|^{*}(\psi)\right)\left(\sigma^{\prime}\right)=\left(|g|^{*}(\psi)\right)\left(\left\langle\sigma^{\prime}\right\rangle\right)=\psi\left(|g|_{*}\left(\left\langle\sigma^{\prime}\right\rangle\right)\right)=\varphi\left(g_{*}\left(\sigma^{\prime}\right)\right),
\end{gathered}
$$

hence the conclusion follows from Lemma 3.2.

\section{Proof of Theorem 1.1}

We can now prove Theorem 1.1 stated in the introduction. We first notice that the construction of $\eta$ is compatible with continuous maps in the following sense.

Lemma 4.1. Let $h: Y \rightarrow Z$ be a continuous map, and let $\mathcal{V}=\left\{V_{i}\right\}_{i \in I}, \mathcal{W}=\left\{W_{i}\right\}_{i \in I}$ be open coverings of $Y, Z$, respectively, such that $h\left(V_{i}\right) \subseteq W_{i}$ for every $i \in I$. The identity of the set $I$ extends to a simplicial map $N(h): N(\mathcal{V}) \rightarrow N(\mathcal{W})$, and in particular it induces a continuous map $\hat{h}:|N(\mathcal{V})| \rightarrow|N(\mathcal{W})|$. Then the following diagram commutes:

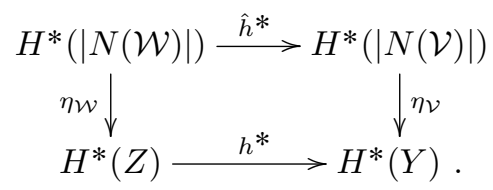


Proof. By considering the restriction of $h$ to the open subset $V_{i}$ the map $h$ induces a morphism $\left\{h^{p, q}\right\}$ between the double complex associated to $\mathcal{W}$ and the double complex associated to $\mathcal{V}$ and between their augmentations. Hence we have $\zeta_{\mathcal{V}} \circ N(h)^{*}=h^{*} \circ$ $\zeta_{\mathcal{W}}: H^{*}(N(\mathcal{W})) \rightarrow H^{*}\left(C_{\mathcal{V}}^{*}\right)$. We also have $\widetilde{\gamma}_{\mathcal{V}} \circ h^{*}=h^{-1, *} \circ \widetilde{\gamma}_{\mathcal{W}}$ and by the definition of the map $\nu$ we have $\nu_{\mathcal{V}} \circ h^{*}=N(h)^{*} \circ \nu_{\mathcal{W}}$. By the definition of $\eta$, these three commutations imply the commutativity claimed in the lemma.

We can now conclude the proof of our main theorem. Let $\mathcal{U}=\left\{U_{i}\right\}_{i \in I}$ be an open covering of the space $X$, let $N(\mathcal{U})$ be the nerve of $\mathcal{U}$, and let $\mathcal{U}^{*}=\left\{U_{i}^{*}\right\}_{i \in I}$ be the open covering of $|N(\mathcal{U})|$ given by the open stars of the vertices of $N(\mathcal{U})$. Let $f_{\Phi}: X \rightarrow$ $|N(\mathcal{U})|$ be the map associated to a partition of unity subordinate to $\mathcal{U}$ as described in the introduction. We would like to apply the previous lemma to the coverings $\mathcal{U}$ of $X$ and $\mathcal{U}^{*}$ of $|N(\mathcal{U})|$ and to the map $h=f_{\Phi}$, but the containment $f_{\Phi}\left(U_{i}\right) \subseteq U_{i}^{*}$ does not hold in general. Therefore, we consider the covering $\tilde{\mathcal{U}}=\left\{\tilde{U}_{i}\right\}_{i \in I}$ of $X$ defined by $\tilde{U}_{i}=f_{\Phi}^{-1}\left(U_{i}^{*}\right)$ for every $i \in I$.

We can now apply Lemma 4.1 to the map $h=f_{\Phi}$ and to the coverings $\mathcal{V}=\tilde{\mathcal{U}}$ and $\mathcal{W}=\mathcal{U}^{*}$. Since $\widetilde{U}_{i} \subseteq U_{i}$ for every $i \in I$, Lemma 4.1 also applies to the case when $h=i_{X}$ is the identity map of $X$, and to the coverings $\mathcal{V}=\tilde{\mathcal{U}}$ and $\mathcal{W}=\mathcal{U}$. Hence we obtain the following commutative diagrams:
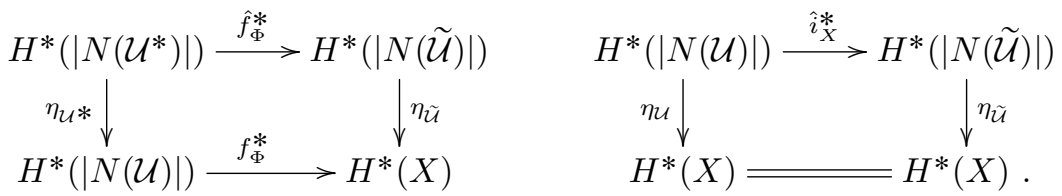

As already noticed in the previous section the simplicial complexes $N(\mathcal{U})$ and $N\left(\mathcal{U}^{*}\right)$ with set of vertices $I$ are equal and, by construction, so are the simplicial maps $N\left(i_{X}\right)$ and $N\left(f_{\Phi}\right)$ from $N(\tilde{\mathcal{U}})$ to $N\left(\mathcal{U}^{*}\right)=N(\mathcal{U})$. In particular $\hat{f}_{\Phi}^{*}=\hat{i}_{X}^{*}$. Finally by Proposition $3.1 \eta_{\mathcal{U}} *$ is the identity. Hence

$$
f_{\Phi}^{*}=f_{\Phi}^{*} \circ \eta_{\mathcal{U}}^{*}=\eta_{\tilde{\mathcal{U}}} \circ \hat{f}_{\Phi}^{*}=\eta_{\tilde{\mathcal{U}}} \circ \hat{i}_{X}^{*}=\eta_{\mathcal{U}},
$$

which proves the theorem.

\section{References}

[Bar95] M. Barr, Oriented singular homology, Theory Appl. Categ. 1 (1995), 1-9.

[BT82] R. Bott and L. W. Tu, Differential forms in algebraic topology, Graduate Texts in Mathematics, vol. 82, Springer-Verlag, 1982.

[ES52] S. Eilenberg and N. Steenrod, Foundations of algebraic topology, Princeton University Press, Princeton, New Jersey, 1952.

[FM] R. Frigerio and M. Moraschini, Gromov's theory of multicomplexes with applications to bounded cohomology and simplicial volume, arXiv:1808. 07307, to appear in Mem. Amer. Math. Soc.

[Gro82] M. Gromov, Volume and bounded cohomology, Inst. Hautes Études Sci. Publ. Math. 56 (1982), 5-99. 
[Hat02] A. Hatcher, Algebraic topology, Cambridge University Pres, Cambridge, 2002.

[Iva] N. V. Ivanov, Notes on the bounded cohomology theory, arXiv:1708.05150.

[Iva87]_, Foundation of the theory of bounded cohomology, J. Soviet. Math. 37 (1987), 1090-1114.

[LS] C. Löh and R. Sauer, Bounded cohomology of amenable covers via classifying spaces, Enseign. Math. 66 (2020), 151-172.

[Wei52] A. Weil, Sur les théorèmes de de Rham, Comment. Math. Helv. 26 (1952), 119-145.

Roberto Frigerio roberto.frigerio@unipi.it

Dipartimento di Matematica, Università di Pisa, Largo B. Pontecorvo 5, Pisa, 56127, Italy

Andrea Maffei andrea.maffei@unipi.it

Dipartimento di Matematica, Università di Pisa, Largo B. Pontecorvo 5, Pisa, 56127, Italy 